\title{
Complex management decisions in a woman with concurrent primary hyperparathyroidism and metastatic papillary thyroid carcinoma, both presenting during pregnancy
}

Correspondence should be addresed to $L$ Arnez

Email

lorena.arnez@nhs.net

\section{Summary}

A 40-year-old woman was hospitalised at 25-week gestation following a diagnosis of severe symptomatic hypercalcaemia (adjusted serum calcium $3.02 \mathrm{mmol} / \mathrm{L}$ ). A diagnosis of primary hyperparathyroidism (PHP) was made on the basis of elevated parathyroid hormone (PTH) $11.2 \mathrm{pmol} / \mathrm{L}$ (reference range 1.5-6.9) and exclusion of familial hypocalciuric hypercalcaemia. Ultrasound examination of the neck did not convincingly demonstrate an abnormal or enlarged parathyroid gland and parathyroid scintigraphy was not performed due to maternal choice relating to perceived radiation risk to the foetus. At neck exploration during the 28th week of pregnancy a right lower pole parathyroid lesion was excised together with two abnormal lymph nodes (largest $1.6 \mathrm{~cm}$ ). Histology confirmed a parathyroid adenoma and also papillary thyroid carcinoma deposits in the two resected lymph nodes. Post-operatively, levels of adjusted serum calcium normalised and pregnancy progressed uneventfully to term. Total thyroidectomy was performed 2 weeks after delivery revealing two small foci of papillary micro-carcinoma (largest $2.3 \mathrm{~mm}$, one in each thyroid lobe) with no evidence of further metastatic tumour in lymph nodes removed during functional neck dissection. Radioiodine remnant ablation (RRA) was performed 2 months post thyroidectomy to allow for breast involution. The patient remains in full clinical and biochemical remission 9 years later. We present and review the difficult management decisions faced in relation to the investigation and treatment of PHP in pregnancy, further complicated by incidentally discovered locally metastatic PT1aN1aM0 papillary thyroid carcinoma.

\section{Learning points:}

- PHP may have serious consequences during pregnancy and usually requires surgical management during pregnancy to reduce the risk of maternal and foetal complications. The indications for and optimal timing of surgical management are discussed.

- Localisation by parathyroid scintigraphy is controversial during pregnancy: modified dose regimes may be considered in preference as an alternative to unguided neck exploration.

- Breastfeeding is contraindicated for 6-8 weeks before radioactive-iodine remnant ablation (RRA) to prevent increased breast uptake. Breastfeeding is further contra-indicated until after a subsequent pregnancy.

- Incidentally discovered differentiated thyroid carcinoma (DTC) in cervical lymph nodes in some cases may be managed expectantly because in one quarter of thyroidectomies the primary tumour remains occult. 


\section{Background}

Synchronous PHP and metastatic papillary thyroid carcinoma (PTC) in pregnancy have rarely been reported (1). To our knowledge, this is the first case of coincidental PHP and metastatic PTC where both conditions were diagnosed in pregnancy. In our case the PTC was multifocal and metastatic and a more aggressive nature of PTC when co-occurring with PHP than PTC without PHP has been suggested (2).

The physiological changes during gestation may mask PHP diagnosis. However, PHP in pregnancy increases the risk of serious complications to both mother and foetus and is difficult to manage because of the paucity of data and limitations on radiological imaging imposed by pregnancy (3).

Surgery for PTC may often be deferred until after delivery depending on stage and growth (4).

\section{Case presentation}

A 40-year-old woman in the 25th week of her third pregnancy was hospitalised and referred to endocrinology services with symptomatic hypercalcaemia after presenting with polydipsia, polyuria and fatigue. Her first pregnancy 20 years previously was uneventful but she had miscarried in the 10th week of gestation shortly prior to the start of her current pregnancy. Relevant family history included neck surgery in a maternal aunt but further information on this has not been possible to obtain.

\section{Investigation}

Blood tests showed an adjusted serum calcium of 3.02 $\mathrm{mmol} / \mathrm{L}$ (reference range (RR): 2.20-2.60), PTH 11.2 pmol/L (RR: 1.5-6.9), phosphate $1.05 \mathrm{mmol} / \mathrm{L}$ (RR: 0.80-1.50), alkaline phosphatase 86 IU/L (RR: 30-95), creatinine $58 \mu \mathrm{mol} / \mathrm{L}$ (RR: 40-90), 25-OH-cholecalciferol $81.9 \mathrm{nmol} / \mathrm{L}$ (WHO adequate $>75$ ). Calcium-to-creatinine clearance ratio was 0.025 which was taken to exclude familial hypocalciuric hypercalcaemia (FHH) in a vitamin D replete individual. Urine calcium excretion was $15.65 \mathrm{mmol} / 24 \mathrm{~h}$. A diagnosis of symptomatic primary hyperparathyroidism was made on the basis of these data.

On examination, blood pressure was $116 / 73 \mathrm{mmHg}$ and heart rate regular at 87 beats per minute. There were no stigmata of multiple endocrine neoplasia or familial hyperparathyroidism syndromes. Neck ultrasonography did not visualise a definite enlarged parathyroid. However, the thyroid showed a diffuse abnormal echo-texture affecting the right lobe without specific features of a discrete nodule and no lymphadenopathy was reported. Antibodies against thyroglobulin and thyroperoxidase were undetectable. Parathyroid scintigraphy was considered and dose calculations suggested that childhood cancer incidence risk might increase from 0.20 to $0.27 \%$ as a consequence of the predicted radiation exposure from the techniques locally available at the time. The patient elected to proceed to unguided bilateral neck exploration without the use of scintigraphy on this basis.

\section{Treatment}

Initial management with intravenous isotonic saline was started. The patient was discharged with an oral intake of $3 \mathrm{~L}$ of fluids daily and was able to maintain a stable adjusted calcium concentration of approximately 2.85 $\mathrm{mmol} / \mathrm{L}$. Discussions were held between endocrinologist, obstetrician, endocrine surgical and anaesthetic specialists and bilateral neck exploration took place in the 28th week of gestation. At operation, the right inferior parathyroid gland was seen to be abnormal and was excised. During the neck exploration two abnormally enlarged lymph nodes (largest $16 \mathrm{~mm}$, one either side of the thyroid bed) were identified and removed for histological examination. The thyroid itself appeared normal at operation and was not therefore resected. Histological examination confirmed the presence of a right parathyroid adenoma and metastatic deposits of follicular variant papillary carcinoma in both of these resected lymph nodes from either side of the neck and a further histological second opinion concurred that the appearances were not those of ectopic thyroid tissue. Post-operatively, adjusted serum calcium concentration normalised (Table 1) and the management of a locally metastatic papillary thyroid carcinoma became the main issue. A functional neck dissection was deemed needed due to PTC deposits on bilateral lymph nodes despite subsequent normal neck ultrasound.

Uneventful spontaneous labour ensued at term and the patient was delivered of a healthy female infant weighing $3.27 \mathrm{~kg}$. Two weeks later, thyroidectomy was performed together with a bilateral functional neck dissection. Histological examination of the total thyroidectomy specimen revealed two foci of papillary carcinoma measuring 2.3 and $1.6 \mathrm{~mm}$ respectively, one focus in each lobe suggesting bilateral multifocal papillary thyroid microcarcinoma. Lymph nodes resected at bilateral functional dissection showed reactive changes with no evidence of further metastases. Therapeutic TSH suppression was commenced, and RRA scheduled 2 months later to 
Table 1 Serum calcium, phosphate, PTH and vitamin D levels at diagnosis of primary hyperparathyroidism, during conservative management and after parathyroidectomy.

\begin{tabular}{l}
\hline \\
\hline Week of gestation \\
Corrected calcium (mmol/L) \\
Phosphate (mmol/L) \\
PTH (pmol/L) \\
25-OH-cholecalciferol (nmol/L)
\end{tabular}

\begin{tabular}{c}
\hline Normal range \\
\hline $2.20-2.60$ \\
$0.80-1.50$ \\
$1.5-6.9$ \\
$>75^{\star}$ \\
\hline
\end{tabular}

\begin{tabular}{c}
\hline At diagnosis \\
\hline 25 th \\
3.02 \\
1.05 \\
11.2 \\
81.9 \\
\hline
\end{tabular}

\begin{tabular}{c}
\hline $\begin{array}{c}\text { During conservative } \\
\text { treatment }\end{array}$ \\
\hline 26 th- 27 th \\
$2.82-2.93$ \\
$1.03-1.05$ \\
NA \\
NA
\end{tabular}

\begin{tabular}{c} 
Post-parathyroidectomy \\
\hline 28 th \\
2.46 \\
1.01 \\
1.9 \\
NA
\end{tabular}

*WHO adequate level.

NA, not applicable.

permit breast involution following cessation of lactation and thereby reduce the exposure of the breasts to radiation. The patient was advised not to restart breastfeeding and the infant was fed with formula milk.

\section{Outcome and follow-up}

Six months after the RRA the thyroglobulin was undetectable in the context of a stimulated TSH of 89.0 $\mathrm{mU} / \mathrm{L}$ (RR: 0.3-5.0). Thyroglobulin antibodies were negative. A radioiodine uptake scan performed 2 years after the surgery showed no evidence of residual or metastatic disease. No complications of PHP have been detected on clinical and imaging follow-up. The patient remains free of recurrence of either PHP or PTC 9 years later.

\section{Discussion}

We report a case where both metastatic papillary thyroid carcinoma (PTC) and primary hyperparathyroidism (PHP) were diagnosed during pregnancy. Although there are a number of reports of PTC and PHP occurring together in pregnancy (1) we believe this to be the first report of metastatic PTC and PHP both being diagnosed during pregnancy.

The diagnosis of hypercalcaemia in pregnancy may be missed or delayed particularly if asymptomatic, and maternal or foetal complications may ensue. Maternal complications include pancreatitis, nephrolithiasis, hyperemesis, muscle weakness, altered mental status, hypercalcaemia crisis and pre-eclampsia. Foetal complications include intrauterine growth restriction, low birth weight, preterm delivery and foetal demise. In the neonate, transient or permanent hypoparathyroidism after delivery may lead to tetany. There is no universal consensus regarding the optimal management of primary hyperparathyroidism during pregnancy. A surgical approach during the second trimester of pregnancy has been recommended for calcaemia above $2.75 \mathrm{mmol} / \mathrm{L}$ on the basis of improved maternal and foetal outcomes (3). Others advocate definitive surgical management at any stage of pregnancy if symptoms are not responding to conservative management or serum calcium remains above $2.9 \mathrm{mmol} / \mathrm{L} \mathrm{(5).} \mathrm{Conservative} \mathrm{treatment} \mathrm{has} \mathrm{been}$ recommended for asymptomatic women with calcaemia below $2.75 \mathrm{mmol} / \mathrm{L}$ as surgery did not improve outcomes in this situation. If conservative management is adopted, intensive foetal and maternal surveillance is essential (5).

During pregnancy, parathyroid adenoma localisation with scintigraphy has historically been avoided to prevent foetal radiation exposure. In our case, calculations suggested that childhood cancer incidence risk might increase from 0.20 to $0.27 \%$ as a consequence of the predicted radiation exposure from the techniques locally available at the time. The ultrasound did not identify an enlarged parathyroid and the patient elected to proceed to unguided bilateral neck exploration without the use of scintigraphy. Offering a 4-gland exploration when preoperative imaging does not identify a single adenoma is recommended by the current national guideline (6). Tc-99m-MIBI has been shown to cross the placenta in animals and is classified as Food and Drug Administration pregnancy category C. For this reason, a dose of Tc-99m-MIBI that causes foetal exposure below $5 \mathrm{mGy}$ has been recommended. $10 \mathrm{mCi}$ of Tc-99m-MIBI, half the standard dose, may be enough to achieve acceptable maternal imaging whilst minimising foetal exposure (7). Increased fluid consumption and urinary voiding may reduce foetal exposure to Tc-99 in the maternal bladder and therefore further limit the foetal absorbed dose. The risks of predicted exposure should be balanced against the potential benefits of more targeted surgery with shorter anaesthetic time and even the potential feasibility of a local-anaesthetic technique that full localisation may permit.

Normal-appearing thyroid follicles in cervical lymph nodes have been found to be present in $4.7 \%$ of autopsies 
(8). These lesions, also called 'nodal thyroid inclusions' or 'ectopic thyroid tissue' are thought to represent dysembryoplasia or 'benign metastasis'. The finding of incidentally discovered PTC in cervical lymph nodes is traditionally considered metastatic and thyroidectomy routinely performed. Recently, a more conservative approach has been advocated by some with longitudinal thyroid imaging assessment. If this shows normal thyroid appearance, then continued follow-up may be a safe option for selected patients (8). The rationale for this approach is that PTC usually has an excellent prognosis and in one quarter of thyroidectomies that follow the discovery of incidental nodal PTC, the primary tumour remains occult (suggested to represent regression of the thyroid tumour or PTC arising within ectopic nodal thyroid tissue). In the present case, bilateral lymph nodes containing metastatic deposits of follicular variant papillary thyroid carcinoma were revealed during neck exploration for PHP. No gross thyroid abnormality was evident intra-operatively so thyroidectomy was not performed at first surgery. Thyroid ultrasound was normal and thyroidectomy showed two microcarcinomas, one in either lobe of the thyroid gland, the largest being $2.3 \mathrm{~mm}$.

When DTC is diagnosed in pregnancy, thyroidectomy during gestation may be considered if there are significant lymph node metastases or rapid DTC growth but generally treatment may safely be deferred until the puerperium (4). In this case, total thyroidectomy and bilateral functional lymph node dissection was performed 2 weeks post delivery.

Breastfeeding leads to increased mammary expression of the sodium-iodide symporter in order to concentrate iodine in milk. This may increase breast irradiation after administration of radio-iodine. Breastfeeding should be withheld for at least 8 weeks and ideally as long as 3 months (9) prior to RRA therapy. If RRA treatment is urgent, I-123 scintigraphy to assess breast uptake may help estimate the likely breast exposure from RRA and dopamine agonists may be used to accelerate breast involution prior to treatment (10).

In conclusion, the management of PHP and metastatic DTC in pregnancy are both challenging. PHP can lead to serious maternal, foetal and neonatal complications and parathyroidectomy is generally recommended during pregnancy, ideally in the second trimester, unless perhaps truly asymptomatic and with adjusted serum calcium levels consistently below $2.75 \mathrm{mmol} / \mathrm{L}$. Parathyroid scintigraphy with a reduced Tc-99m dose may be considered during pregnancy and radiation risks to the foetus weighed against the benefits of successful pre-operative localisation.
Surgical management of DTC can usually be safely delayed until after delivery. Breastfeeding should be stopped 8 weeks before RRA to reduce breast radiation risk.

\section{Declaration of interest}

The authors declare that there is no conflict of interest that could be perceived as prejudicing the impartiality of this case report.

\section{Funding}

This research did not receive any specific grant from any funding agency in the public, commercial or not-for-profit sector.

\section{Patient consent}

Written informed consent has been obtained from the patient for publication of the submitted article.

\section{Author contribution statement}

$\mathrm{L}$ Arnez wrote the case report. $\mathrm{V}$ Lawrence was responsible for the case management and co-authored the article.

\section{References}

1 Baumann K, Weichert J, Krokowski M, Diedrich K \& Banz-Jansen C. Coexistent parathyroid adenoma and thyroid papillary carcinoma in pregnancy. Archives of Gynecology and Obstetrics 2011284 91-94. (https://doi.org/10.1007/s00404-011-1903-0)

2 Çetin K, Sıkar HE, Temizkan Ş, Ofluoğlu CB, Özderya A, Aydın K, Gül AE \& Küçük HF. Does primary hyperparathyroidism have an association with thyroid papillary cancer? A retrospective cohort study. World Journal of Surgery 201943 1243-1248. (https://doi. org/10.1007/s00268-019-04920-4)

3 Norman J, Politz D \& Politz L. Hyperparathyroidism during pregnancy and the effect of rising calcium on pregnancy loss: a call for earlier intervention. Clinical Endocrinology 200971 104-109. (https://doi.org/10.1111/j.1365-2265.2008.03495.x)

4 De Groot L, Abalovich M, Alexander EK, Amino N, Barbour L, Cobin RH, Eastman CJ, Lazarus JH, Luton D, Mandel SJ, et al. Management of thyroid dysfunction during pregnancy and postpartum: an Endocrine Society clinical practice guideline. Journal of Clinical Endocrinology and Metabolism 201297 2543-2565. (https:// doi.org/10.1210/jc.2011-2803)

5 Schnatz PF \& Curry SL. Primary hyperparathyroidism in pregnancy: evidence-based management. Obstetrical and Gynecological Survey 2002 57 365-376. (https://doi.org/10.1097/00006254-200206000-00022)

6 Recommendations. Hyperparathyroidism (primary): diagnosis, assessment and initial management. Guidance NICE. (available at: https://www.nice.org.uk/guidance/ng132/chapter/Recommendatio ns\#non-surgical-management)

7 McMullen TPW, Learoyd DL, Williams DC, Sywak MS, Sidhu SB \& Delbridge LW. Hyperparathyroidism in pregnancy: options for localization and surgical therapy. World Journal of Surgery 201034 1811-1816. (https://doi.org/10.1007/s00268-010-0569-2)

8 Triantafyllou A, Williams MD, Angelos P, Shah JP, Westra WH, Hunt JL, Devaney KO, Rinaldo A, Slootweg PJ, Gnepp DR, et al. Incidental findings of thyroid tissue in cervical lymph nodes: old controversy not yet resolved? European Archives of 
Oto-Rhino-Laryngology 2016273 2867-2875. (https://doi. org/10.1007/s00405-015-3786-3)

9 Azizi F \& Smyth P. Breastfeeding and maternal and infant iodine nutrition. Clinical Endocrinology 200970 803-809. (https://doi. org/10.1111/j.1365-2265.2008.03442.x
10 Brzozowska M \& Roach PJ. Timing and potential role of diagnostic I-123 scintigraphy in assessing radioiodine breast uptake before ablation in postpartum women with thyroid cancer: a case series. Clinical Nuclear Medicine 200631 683-687. (https://doi. org/10.1097/01.rlu.0000242600.23386.1a)

Received in final form 8 November 2019

Accepted 22 November 2019 